\title{
A HISTÓRIA DA MÚSICA DE RIBEIRÃO PRETO (SP) ATRAVÉS DE ARQUIVOS DE MÚSICOS E CORPORAÇÕES
}

\section{THE HISTORY OF MUSIC OF RIBEIRÃO PRETO (SP) THROUGH ARCHIVES OF MUSICIANS AND CORPORATIONS}

\author{
Gisele Laura Haddad \\ Universidade de São Paulo \\ gisele.haddad@usp.br
}

\begin{abstract}
Resumo
Durante o processo da investigação para o mestrado "Orquestra Sinfônica de Ribeirão Preto: Representações e Significado Social" (HADDAD, 2009), localizamos diversos arquivos pessoais de músicos, exmúsicos e corporações de Ribeirão Preto. Com os entraves de acesso superados, verificamos que muito da história da cidade está com a população, dentro das casas, nos álbuns de familia, nas cartas e recortes de jornais, documentação pessoal, de imprensa, iconográfica, bem como manuscritos e impressos musicais entre outros documentos, muitos deles até então inexplorados. Este artigo apresenta o projeto de doutorado que tem como objetivo principal o levantamento de fontes históricas das atividades musicais, desde a fundação da cidade em 1856 até meados da década de 1980, através dos acervos pessoais dos acervos de: Aluísio da Cruz Prates (1931), Belmácio Pousa Codinho (1892-1980), Conegundes Rangel, Edmundo Russomanno (1893-1963), Francisco de Assis Barros, também conhecido como Cônego Barros (1894-1942), Franklin Guedes Carneiro Filho, Gaetano Baccega, Homero de Sá Barreto (1884-1924), Ignácio Stábile (18891955), José Delfino Machado, José dos Reis Miranda Filho (1901-1956),
\end{abstract}


José Luiz Baldo (1909-2010) e Manoel da Silva (1896-1963). Tais acervos e demais documentos relativos à história da música de Ribeirão Preto estão depositados em residências da cidade e nos arquivos locais (Arquivo do Museu do Café de Ribeirão Preto, Centro de Documentação "Memória Musical Brasileira" da FFCLRP/USP, Departamento de Música da UNAERP, Arquivo Histórico da Orquestra Sinfônica de Ribeirão Preto e Arquivo Público Histórico Municipal de Ribeirão Preto). Para esta pesquisa são considerados aspectos histórico-musicológicos em suas evidentes implicações culturais e sociais. $\bigcirc$ levantamento de todos esses dados abre caminho para a compreensão da movimentação musical como característica dos significados sociais que a música exercia, dando parâmetros para o estudo da história da música local. Por conseguinte, estabelecemos conceitos para a discussão teórica: o conjunto de caracteristicas e circunstâncias sociais que distinguem a sociedade e a tradição musical da cidade de Ribeirão Preto.

Palavras-chave: arquivos; centro de documentação; Ribeirão Preto

\begin{abstract}
During the research process for the master's "Symphonic Orchestra of Ribeirão Preto: Representations and Social Meaning" (HADDAD, 2009), located several personal files of musicians, former musicians and Ribeirao Preto corporations. With overcome access barriers, we find that much of the city's history is with the people, inside homes, in family albums, the letters and newspaper clippings, personal documents, press, iconographic, as well as manuscripts and musical printed between other documents, many of them hitherto unexplored. This article presents the $\mathrm{PhD}$ project that aims raising historical sources of musical activities, since the city's founding in 1856 until the mid-1980s, through the personal collections of collections: Aluísio da Prates Cruz (1931), Belmácio Pousa Godinho (1892-1980), Conegundes Rangel, Edmundo Russomanno (1893-1963), Francisco de Assis Barros, Cônego Barros (1894-1942),
\end{abstract}


Franklin Guedes Carneiro Filho, Gaetano Baccega, Homero de Sá Barreto (1884-1924), Ignacio Stábile (1889-1955), José Delfino Machado, José dos Reis Miranda Filho (190 l - 1956), José Luiz Baldo (1909-20 10) and Manoel da Silva (1896 to 1963). Such archives and other documents related to the history of Ribeirão Preto music are deposited in the city's homes and in local files (File Museum of Ribeirão Preto Café, Documentation Centre "Brazilian Musical Memory" of FFCLRP / USP, Music Department UNAERP, Historical Archive of the Symphonic Orchestra of Ribeirão Preto Public and Municipal Historical Archive of Ribeirão Preto). For this survey are considered historical and musicological aspects of its obvious cultural and social implications. The survey of all this data opens the way to understanding the musical movement as a characteristic of social meanings that music exercised, giving parameters for the study of the history of local music. Therefore, we established concepts to theoretical discussion: the set of characteristics and social circumstances that distinguish the company and the musical tradition of the city of Ribeirõo Preło.

Keywords: archives; documentation centre; Ribeirão Preto

\section{Introdução}

Em 2006, por sugestão do Prof. Dr. Marcos Fernandes Pupo Nogueira do Instituto de Artes da Universidade Estadual Paulista "Júlio de Mesquita Filho" (IA-UNESP/SP), surgiu o projeto de mestrado sobre a Orquestra Sinfonica de Ribeirão Preto. Inicialmente o que se pretendia era a investigação da vida e obra de três compositores locais; Ignácio Stábile (1889-1955), Homero de Sá Barreto (1 884-1924) e Belmácio Pousa Godinho (1892-1980), cujas documentações musicais supostamente estariam no Arquivo Histórico da Associação Musical mantenedora da orquestra, conhecido como Arquivo Histórico da Orquestra Sinfônica.

No mesmo ano, manuscritos, fotos, recortes de jornais e progra- 
mas de concertos foram localizados nesse arquivo sobre o maestro lgnácio Stábile. Pouca documentação existia sobre Homero de Sá Barreto e apenas a menção ao nome de Belmácio Pousa Codinho em alguns programas de concertos. Ao mesmo tempo, havia a necessidade de se investigar a existência da orquestra na cidade, até então considerada por ela mesma como uma das mais antigas do país em funcionamento ininterrupto e também justificar como em Ribeirão Preto, cidade do interior do pais, que aparentemente não teria precedentes da música erudita, uma orquestra sucumbiu ao tempo. O projeto mudou e em 2009 foi concluída a dissertação de mestrado pelo IA- UNESP/SP sob orientação do Prof. Dr. Paulo Augusto Castagna: Orquestra Sinfônica de Ribeirão Preto: Representações e Significado Social, publicada em 2011 pelo Instituto do Livro de Ribeirão Preto, em parceria com a Secretaria Municipal da Cultura e Câmara Municipal de Ribeirão Preto, pelo concurso: Prêmio Coleção Nossa História.

Durante o processo da investigação para o mestrado, além da pesquisa no Arquivo Público Histórico Municipal de Ribeirão Preto e do Arquivo Histórico da Orquestra Sinfônica, localizamos os arquivos pessoais do violoncelista ex-músico da orquestra, Manoel da Silva (1896-1963), do violinista Luiz José Baldo (1909-20 10) e do clarinetista Aluísio da Cruz Prates (1931). Com exceção do arquivo de Aluísio, os entraves para o acesso aos arquivos pessoais foram muitos; outros arquivos pessoais de músicos possuem álbuns fotográficos de familia e documentos que registraram a atividade musical em Ribeirão Preto, principalmente do início do século XX, mas logo nos deparávamos com a desconfiança e alguns ransos dos músicos, já em idade avançada, em relação ao seu desligamento orquestra que foram fundadores. Portas fechadas. Dificuldades foram diminuídas com a intercessão de amigos em comum; Eva Fachin Galeotte, que, como católica e ministra da eucaristia, convidou-me para acompanhá-la e levar a hóstia na casa de Luiz Baldo, e de Nivaldo Laguna Ciocchi, primo da familia de Manoel da Silva.

Posteriormente, em 2013, no levantamento de dados para este 
projeto de doutorado, foi localizado o arquivo pessoal do violinista José dos Reis Miranda Filho (1901-1956), de propriedade de sua neta Dirce Maria Miranda Ribeiro. Nele contém um recorte de jornal que comprova o que os estudos anteriores apontavam como indícios; a orquestra Sinfônica de Ribeirão Preto foi fundada em 1921. Disso, voltamos à constatação: muito da história de Ribeirão Preto está com a população, dentro das casas, nos álbuns de familia, nas cartas e recortes de jornais entre outros documentos. Está nos álbuns de familia, sempre com o risco da ideia e do discurso: "bem, agora que seu avô se foi, vamos nos livrar daquela papelada velha que ele juntava", por falta de conhecimento ou desconsideração. Logo vem a indagação: quanto já não foi descartado? A desconfiança afasta as pessoas e é realmente dificil a disposição das pessoas em abrir os arquivos e a intimidade familiar a estranhos. E o destino fica nas mãos da abnegação dos estudantes e pesquisadores nessa busca, nos seus objetivos em preservar a história.

\section{Centralização de acervos musicais de Ribeirão Preto e região}

$\mathrm{Na}$ preocupação com o destino da documentação musical em posse das familias de músicos e compositores, o professor titular do Departamento de Música da FFCLRP-USP, Rubens Russomanno Ricciardi, idealizou o Centro de Documentação Memória Musical Brasileira (CEDOC-MMB) do Departamento de Música da Faculdade de Filosofia Ciências e Letras da USP de Ribeirão Preto, para centralizar os arquivos, muitos dos quais ele mesmo conseguiu a doação de pessoas de Ribeirão Preto e região para o estudo e pesquisa. $\bigcirc$ projeto foi apresentado aos Programas Especiais \& Editais 2012 da Pró-Reitoria de Cultura e Extensão Universitária da Universidade de São Paulo e contemplado para a compra de equipamentos específicos para o armazenamento, manutenção e difusão do acervo.

Desde 2002, várias coleções (manuscritos e impressos musicais, 
fontes iconográficas, hemerográficas e demais documentos e objetos) de músicos, compositores e corporações musicais foram doadas ao Curso de Música da USP. Provisoriamente, estas coleções foram guardadas na Biblioteca Central do Campus da USP de Ribeirão Preto (em sala de acesso restrito). $\bigcirc$ Prof. Dr. Rubens Russomanno Ricciardi iniciou o trabalho de organização destas coleções, viabilizando, para esta finalidade, dois trabalhos de Iniciação Científica de alunos de graduação em música pelo Campus da USP de Ribeirão Preto, Juliana Damaris Santana (Catalogação dos acervos de Bandas da Região de Ribeirão Preto, 2005, com bolsa da FAPESP) e Lucas Eduardo da Silva Calon (Catalogação dos acervos Belmácio Pousa Codinho, Edmundo Russomano e Caetano Baccega, 2005, com bolsa do CNPa).

A partir de agosto de 2012 , após inauguração do Bloco 34, que sedia o Departamento de Música da FFCLRP-USP, a Tulha e seus anexos receberam o Núcleo de Pesquisa em Ciencias da Performance e este Centro de Documentação. Há nisso o cuidado constante em se manter um organismo integrador de fontes da música brasileira, numa atividade indissociável da pesquisa histórico-musicológica a partir de fontes primárias, proporcionando a devida manutenção, organização e difusão de seu acervo.

\section{Pesquisas e publicações recentes}

Em 2011 ingressei como docente no Curso de Licenciatura em Música da Universidade de Ribeirão Preto (UNAERP) e pude orientar 
alguns trabalhos de conclusão de curso' de cunho histórico-musicológico sobre Ribeirão Preto e região, bem como ter acesso ao arquivo musical da instituição que está alocada no Departamento de Música, onde funciona o curso.

Diante das lacunas existentes sobre a história da música de Ribeirão Preto, verificamos que existem poucas publicações sobre o tema; como o livro A Música em Ribeirão Preto: Manifestações do começo do século XX de Thaty Mariana Fernandes (20 1 1), a dissertação sobre a Orquestra Sinfônica já aqui citada, o livro 50 Anos de Orquestra Sinfonica em Ribeirão Preto da memorialista Myriam de Souza Strambi (1988) e a dissertação de mestrado Belmácio Pousa Godinho: vida e obra do compositor paulista, concluida em 2008 na Escola de Comunicações e Artes da Universidade de São Paulo (ECA-USP/SP) por Gustavo Augusto Molinari. Outra referência é a monografia de José Pedrosa Ferraz Júnior, intitulada A Criação da Orquestra Sinfônica na Ribeirão Preto dos anos de 1930 (2007), que mais discorre acerca da vida e obra de Max Bartsch, seu primeiro presidente. Foi com Ferraz

'MONTEIRO, Cesar Augusto de Paula. Ze da Conceição: Recortes de um universo Musical. 201 1. Trabalho de Conclusão de Curso. (Craduação em Licenciatura em Música) - Universidade de Ribeirão Preto. Orientador: Cisele Laura Haddad.

CUNHA, Renata Baldon. Diva Tarlá de Carvalho: Um Resgate Histórico. 2011 . Trabalho de Conclusão de Curso (Craduação em Licenciatura em Música) - Universidade de Ribeirão Preto. Orientador: Cisele Laura Haddad.

SANTOS, Marcelo Cosme dos. Banda Musical de Concerto de Santa Cruz das Palmeiras: Um Relato de Experiência. 2012. Trabalho de Conclusão de Curso (Craduação em Licenciatura em Música) - Universidade de Ribeirão Preto. Orientador: Cisele Laura Haddad.

SANCHIN, Ligia Dardani. Nascimento e Consolidação do Coral da USP do Campus de Ribeirão Preto. 2012. Trabalho de Conclusão de Curso. (Craduação em Licenciatura em Música) - Universidade de Ribeirão Preto. Orientador: Gisele Laura Haddad.

ANDRÉ, Érika Fernandes. O Maestro Italiano: Atuação do Maestro Ignázio Stábile em Ribeirão Preto de 1923 a 1955. 2012. Trabalho de Conclusão de Curso. (Craduação em Licenciatura em Música) - Universidade de Ribeirão Preto. Orientador: Gisele Laura Haddad.

MARQUES Isabela Meni Cosenza. Myrian de Souza Strambi - Produção Literária e Pedagógico-Musical. 20 13. Trabalho de Conclusão de Curso (Craduação em Licenciatura Plena em Música) - Universidade de Ribeirão Preto. Orientador: Gisele Laura Haddad. 
que publiquei em 2013 o livro comemorativo Jubileu de Brilhante - Os 75 anos da Associação Musical de Ribeirão Preto, na união de nossos trabalhos acadêmicos sobre a Orquestra Sinfônica e pesquisas mais recentes. Outros poucos estudos que relatam a vida social e cultural de Ribeirão Preto no começo do século XX são encontrados nas teses e dissertações do Departamento de História da UNESP de Franca.

Em vista desta escassez, esta tese tem como objetivo principal - levantamento de fontes históricas das atividades musicais da cidade de Ribeirão Preto, entre sua fundação em 1856 chegando a meados da década de 1980, através dos acervos pessoais de músicos e compositores, muitos deles até então inexplorados que estão localizados no Arquivo do Museu do Café de Ribeirão Preto, no recém-fundado Centro de Documentação "Memória Musical Brasileira" (CEDOC-MMB) da Faculdade de Filosofia Ciências e Letras da USP de Ribeirão Preto (FFCLRP/USP), no Departamento de Música da Universidade de Ribeirão Preto (UNAERP), no Arquivo Histórico da Orquestra Sinfônica de Ribeirão Preto e no Arquivo Público Histórico Municipal de Ribeirão Preto. Portanto, o que se soma aos arquivos pessoais de Manoel da Silva de propriedade de sua filha Maria de Lourdes Silva Laguna, de Luiz José Baldo, de propriedade de sua filha Maria Lúcia Baldo carvalho e de Aluísio da Cruz Prates, investigados durante o mestrado sobre a Orquestra Sinfonica, são documentações localizadas no CEDOC-MMB referentes aos compositores Belmácio Pousa Codinho, Conegundes Rangel, Edmundo Russomanno, Francisco de Assis Barros (Conego Barros), Franklin Guedes Carneiro Filho, Gaetano Baccega, Homero de Sá Barreto e José Delfino Machado e registros relativos à atividade musical de Ribeirão Preto e região, como as bandas municipais e a banda Santa Cecilia que se apresentava no bosque municipal, extinta em meados da década de 1980. Esta última, assim como a orquestra, era mantida por uma associação. Localizamos isoladamente também a se somar a esses arquivos pessoais, o álbum de familia do violinista José dos Reis Miranda Filho, de propriedade de sua neta Dirce Maria Miranda Ribeiro, o arquivo de Ignácio Stábile de propriedade do Arquivo Histórico da Orquestra. 


\section{Música e sociedade}

O levantamento de todos esses dados abre caminho para a compreensão da movimentação musical como característica dos significados sociais que a música exercia, dando parâmetros para o estudo da história da música local. Por conseguinte, estabelecemos conceitos para a discussão teórica: o conjunto de características e circunstâncias sociais que distinguem a sociedade e a tradição musical da cidade de Ribeirão Preto.

Trabalhamos com a hipótese de que os acervos pessoais juntos são uma amostra abrangente das produções musicais realizadas em Ribeirão Preto, evidenciando sua história e sociedade e colocando este cenário na história musical do país. Trata-se também de um levantamento sobre o repertório utilizado no periodo ( 1856 - 1980) por esses músicos e corporações, demonstrando sua renovação, observando até onde se contempla os compositores brasileiros e/ou locais e se existem arranjos buscando entender as expectativas do público dentro do panorama histórico regional e nacional. Estes tópicos são considerados e analisados, buscando na música de Ribeirão Preto deste período não apenas a recuperação da história regional, mas a preservação histórica musical brasileira.

Também pretendemos analisar, dentro do período proposto, alguns aspectos referentes ao repertório da Orquestra Sinfônica de Ribeirão Preto e dos conjuntos musicais que atuavam na cidade como: estilo da época atrelado ao repertório de banda, ou, por outro lado, sua dinâmica de renovação, sintonia com o neofolclorismo ou futurismo, ou ainda interfaces com os primórdios da indústria da cultura.

Esta pesquisa é parte de um movimento que se observa em Ribeirão Preto através dos tempos; o de contar a sua história, afirmar sua identidade, características e tradições, independente do eixo Rio-São Paulo. 
Este estudo poderá ser inserido nas pesquisas que atualmente acontecem no Departamento de Música da USP em Ribeirão Preto considerando que os outros acervos pessoais não constantes no CEDOC -MMB mostram-se inéditos e sua análise critica mostrará em que ele se assemelhará ou se diferenciará dos trabalhos dos outros compositores já citados dentro do mesmo cenário musical.

método adotado, tendo em vista os objetivos estabelecidos, é o histórico-analítico, que orientou na leitura e interpretação dos elementos fundamentais para a elaboração do trabalho. Através de trabalho bibliográfico acerca do tema e com o estudo e análise da bibliografia, buscamos a reflexão e aprofundamento, mediante 0 confronto de ideias e informações para, a seguir, empreender pesquisa documental, utilizando-se como fontes os documentos existentes nos arquivos, relatórios, programas de apresentações musicais e artísticas da cidade de Ribeirão Preto.

Com a análise de depoimentos, livros, peças musicais e outras fontes de pesquisa, contidos nos arquivos da cidade, procuramos compreender a relevância das primeiras bandas a atuarem na cidade e posteriormente da Orquestra Sinfônica (OSRP) para a sociedade ribeirãopretana e da região, bem como a evidência dos principais músicos e compositores da época (segunda metade do século XIX e século XX) e sua relação com os repertórios e eventos do período, numa continuidade das pesquisas realizadas por ocasião da dissertação de mestrado intitulada Orquestra Sinfônica de Ribeirão Preto: Representações e Significado Social (HADDAD, 2009).

\section{Considerações finais}

Através dos arquivos pessoais confrontamos os valores históri$\cos e$ artísticos e nesse confronto de ideias e informações resultamos num maior entendimento sobre repertório, músicos e compositores e a sua relação para com a época e a sociedade onde a atividade mu- 
sical sinfônica e das bandas de coreto se insere.

Além, disso, é preciso estar convencido da importância da natureza do cânone social utilizado em Ribeirão Preto desde seus primórdios; de sua mutabilidade ao longo da história e da necessidade de fazermos sempre uma crítica dele a fim de revelar outros pensamentos e outros autores. Não podemos nos limitar a aceitar as regras padrões estabelecidas sem ter o senso crítico para buscar as razões que levaram este e não aquele compositor a servir de suposto modelo a este trabalho. Ginzburg (2012) é um dos autores que colaboram para esta fundamentação térica, pois apesar de escrever sobre literatura, a analogia com o histórico-musical acontece na reflexão que articula texto e contexto, dentro das perspectivas interdisciplinares.

O levantamento bibliográfico para o estudo dos compositores e atuação dos músicos em questão está ancorado na sociologia e na antropologia estabelecendo-se caminho para uma primeira abordagem, explicando as razões que os levaram a serem compositores e músicos. Bourdieu (1989) formula importantes referencias quanto à legitimação dos grupos musicais perante a sociedade e o trabalho dos músicos e compositores. Portanto, o panorama histórico da região de Ribeirão Preto nos oferece a coerência para o entendimento e as reflexões. Caracterizamos o cenário social, econômico e musical da cidade na segunda metade do século XIX e na primeira metade do século XX, com base nos estudos e pesquisas de âmbito nacional, no campo da musicologia histórica. Para tanto, Raynor (1981) é considerado ao elucidar que a história não é apenas um registro cronológico dos fatos, mas sim uma elucidação do passado através do presente e vice-versa. Por isso mesmo, não deixamos de tratar em reflexões sobre as questões referentes à movimentação cultural em Ribeirão Preto no século XXI, como herança de seu passado e analisando as condições de como é aplicada a lei Rovanet para projetos culturais na cidade. 


\section{Referências}

BOURDIEU, Pierre. A Economia das trocas simbólicas. São Paulo: Perspectiva, 1987.

BOURDIEU, Pierre. O poder simbolico. Rio de Janeiro: Bertrand Brasil, 1989.

CIONE, Rubem. História de Ribeirão Preto. Matão: IMAG, 1987.

COSTA, Ordones da; HADDAD, Gisele Laura. "P.R.A.-7, a Rádio do Coração do Brasil: repercussão no cenário musical de Ribeirão Preto (SP)". In Ictus, Salvador: v. 9, p. 73-88, 2008.

DAVID, Célia Maria. Criação e interpretação musical em Franca: palco e plateia (1872-1964). Franca: UNESP/FHDSS, 2002.

FERNANDES, Thathy Mariana (pesquisa e texto). A música em Ribeirão Preto - manifestações do começo do século XX. Coleção Identidades Culturais, n.6. Ribeirão Preto: Fundação Instituto do Livro, 2011.

GINZBURG, Jaime. Crítica em tempos de violência. São Paulo: EDUSP/ FAPESP, 2012.

HADDAD, Gisele Laura, FERRAZ, Jr. Jubileu de Brilhante: Os 75 anos da Associação Musical de Ribeirão Preto - 1.ed. Ribeirão Preto, SP: Editora Corvja, 2013.

HADDAD, Gisele Laura. Orquestra Sinfônica de Ribeirão Preto: Representações e Significado Social - 2009, 109 f. dissertação de Mestrado Instituto de Artes Universidade Estadual Paulista Júlio de Mesquita Filho, São Paulo, 2009.

RAYNOR, Henry. Historia social da música. Da ldade Média a Beethoven. Tradução de Nathanael C. Caixeiro. Rio de Janeiro: Editora Guanaba- 
ra, 1981.

RICCIARDI, Rubens Russomano. Centro de Documentação "Memória Musical Brasileira". Projeto apresentado aos Programas Especiais \& Editais 2012 da Pró-Reitoria de Cultura e Extensão Universitária da Universidade de São Paulo. Faculdade de Filosofia, Ciências e Letras de Ribeirão Preło: 2012.

SMALL, Christopher. "The social character of music. Performance as ritual: sketch for an enquiry into the true nature of a symphony concert". In Sociological review monograph 34. Lost in Music: Culture, Style and Musical Evente. Edited by Avron Levine White. London and New York, Routledge \& Kegan Paul, 1987.

STRAMBI, Miriam. 50 anos de Orquestra Sinfônica em Ribeirão Preto. Ribeirão Preto: Legis Summa, 1989. 